\title{
Economic Benefit Evaluation Model of Urban Street Reconstruction Based on Parallel Multigrid Computing
}

\author{
Yu Cui \\ School of Art, Xi'an Fanyi University, Xi'an, Shannxi 710105, China \\ Correspondence should be addressed to Yu Cui; cuiyu@xafy.edu.cn
}

Received 20 December 2021; Revised 14 January 2022; Accepted 26 January 2022; Published 18 February 2022

Academic Editor: Sheng Bin

Copyright (c) $2022 \mathrm{Yu}$ Cui. This is an open access article distributed under the Creative Commons Attribution License, which permits unrestricted use, distribution, and reproduction in any medium, provided the original work is properly cited.

\begin{abstract}
This study draws on the relevant experience of algebraic multigrid, which effectively deals with anisotropic matrices, transforms the geometric characteristics of unstructured grids into numerical relationships, distinguishes the degree of anisotropy of geometric grids through numerical values, and forms a strong-weak coupling relationship between elements. It is used to aggregate and generate coarse grid levels, which makes up for the defect that the cell-centric calculation format cannot effectively deal with high-ductility grids. In order to reduce the computational complexity of the coarse-grid layer and adapt to the calculation requirements of different dimensions, multiple strong coupling aggregations are used for high-ductility grid cells and cell-centric aggregations are used for other grid cells. Under the RGD model, the government pays attention to the improvement of environmental quality and land fiscal revenue. Residents pay attention to the level of demolition compensation and resettlement fees and the improvement of urban landscape functions. Developers pay attention to urban renewal and renovation costs, public participation, and coordination between new and old buildings. The government pays attention to the level of demolition compensation and resettlement fees and the cost of urban renewal and transformation, residents are concerned about the improvement of cultural education and the level of rental income, and developers are concerned about the improvement of traffic conditions, land utilization, and urban landscape functions. The government pays attention to the level of demolition compensation and resettlement fees and the status of land fiscal revenue, residents pay attention to public participation, and developers pay attention to land use. The comprehensive benefit evaluation of urban renewal is multidimensional, and comprehensive evaluation is carried out from the six dimensions of the social, economic, and environmental perspectives of the government, residents, and developers that coordinate the benefits and the social, economic, and environmental aspects of the development benefits. Social and people's livelihood, economic development, environmental support, ecological balance, and historical and cultural inheritance are the core content of the evaluation system. The social and people's livelihood is the foundation, economic development is an important condition, the environment is an important factor, and history and culture are the connotations. Urban renewal is not only about economic development but more concerned about the sustainable development of the society and the environment. Due to many factors, before starting an urban renewal project, the implementation plan should be comprehensively evaluated from multiple perspectives and dimensions and the plan and investment scale should be reasonably formulated to achieve the best goal of sustainable development.
\end{abstract}

\section{Introduction}

The street is an important element of the city. It can help people walk through the streets and alleys to reach their destinations. They are distributed in the area around the street. The streets are enclosed together to form a large and small shared communication space. Sometimes the function of the street is single, and sometimes it is integrated. The existence of a city is largely due to social activities [1]. The street is a place where people gather and communicate and is a space for walking. At the same time, the street itself is also viewed by people as a kind of landscape. Urban design is an effective means to improve the overall urban spatial environmental quality. It controls the urban spatial structure, spatial characteristics, skyline, visual corridors, etc. at the macro level and controls and guides the design elements 
such as architectural color, material, height, and volume at the micro level [2]. Therefore, as one of the achievements of urban design, the urban design guidelines have become an important means to shape urban space, supplement control, and guide content [3]. The subject of modern urban design has become an interdisciplinary subject linking urban and rural planning, architectural design, landscape architecture, and other subjects, and it can solve the planning and design problems of urban three-dimensional space in a targeted manner [4]. Urban design can make up for the control of the specific space in the city. The shortcomings of construction management and characterization also make up for the shortcomings of urban and rural planning in terms of spatial design, which only focuses on two-dimensional land use. Therefore, urban design acts as a "shock absorber" between the use of two-dimensional land and the construction of three-dimensional urban space.

Based on the many problems in the old city, the urban design guidelines are used as a strategic means, and based on the special regional background inside the old city and the public's suggestions for the planning and construction of the old city, the design elements of the old city's urban design guidelines are selected and approved [5]. Different levels of management implement the urban design guidelines. In this reasoning process, the key points of the compilation of the urban design guidelines for the old city are explored and a guide system is formed, providing a scientific and effective method for the development of the old city. From the perspective of a relatively specific solution to the actual needs of street space development, the coordinated development of street space, the surrounding environment, and various influencing factors of the city will help improve the city's cultural outlook and urban characteristics [6]. The introduction of rational thinking and perceptual design into the street space design of West 11th Road in Mudanjiang gave us a brand-new perspective and level to carry out in-depth discussions on the issue of its renewal and development and to put forward corresponding specific countermeasures [7]. Renovation design has certain guidance and inspiration and summed up universal street design principles and methods. Through in-depth analysis of the connotation of rational thinking, perceptual design, and the feasibility of space reconstruction in the street space of West 11th Road in Mudanjiang, it provides a practical reference for the construction of a comprehensive landscape system of urban streets. The update provides a new development model and proposes new theories and practical exploration experience for the development of the street space connecting the new and old urban areas. In addition, urban design is more scientific and reasonable to coordinate, take into account, and control the entire urban space environment and give priority to shaping the urban spatial form than the detailed construction planning or architectural design in a single project or plot. Therefore, the urban design guidelines have a positive auxiliary guiding role in improving the spatial quality of the old city.

In this study, aiming at the GMG method of the cellcentric calculation format, when dealing with anisotropic grids, the problem of low convergence efficiency is analyzed.
The node-centric calculation format and related processing techniques in AMG are analyzed, and the correlation of nonsmooth aggregation in AMG is learned. The research results transform the anisotropic geometric characteristics of unstructured grids into numerical relationships, distinguish the strong and weak geometric coupling relationships by numerical values, and develop a strong coupling aggregation strategy. Some assumptions are made on the strategic choices of urban renewal stakeholders and the factors and conditions that affect the overall benefits, and their authenticity is tested through formula derivation, combined with theoretical analysis and qualitative analysis. According to the comprehensive benefit evaluation model of urban renewal, the fuzzy mathematics method is applied to comprehensively evaluate the results of three projects, and the comprehensive benefit value is quantified by the improved radar chart method. Combined with the comprehensive benefit evaluation chart of urban renewal, it is shown that the comprehensive benefits of the three projects belong to basic benefit basic coordinated development projects, which are more in line with actual conditions. The comparative analysis shows that the comprehensive benefit value of street renovation in a certain urban area is the highest, indicating that the government will auction land on behalf of the government to obtain renovation funds. The RGD model where residents complete the renovation is better than the GRD model where the government is led by administrative means, and the development is completely funded and led by the developer. The DGR model is more able to balance the interests of all parties, and the overall benefits are higher.

1.1. Related Work. Information exchange between grids is another key issue in the overlapping grid method, which has an important impact on the calculation of the flow field. If not properly handled, it will not only reduce the convergence speed of the flow field solution but also cause the appearance of nonphysical understanding. In the overlapping grid method, the information exchange between grids is generally achieved through interpolation between grids, and interpolation will cause the physical quantity of the flow field to be unconserved [8]. Therefore, after the concept of overlapping grids was put forward, the results obtained by nonconservative interpolation are not credible.

In the dynamic unstructured grid method, if the displacement of the moving boundary is small, such as an aeroelasticity problem, the above dynamic deformation method can be appropriately selected. However, in the simulation of the flow around the multibody with large relative motion, the above method alone cannot meet the needs. In this case, the method of mesh deformation and local mesh regeneration is generally used. In the dynamic mesh method combining mesh deformation and local mesh regeneration, the mesh first uses one of the above models to deform with the moving object. When the quality of the deformed mesh cannot meet the requirements, such as negative volume, the mesh is regenerated for the local area 
near the moving object. Finally, data transfer from the old grid to the new grid is achieved by interpolation.

Relevant scholars used the object surface distance and the grid cell size as the criterion parameters in the definition of the boundary between the grids of the unstructured overlapping grid, which not only fully considered the influence of the object surface on the flow field but also considered the grid cell size on the calculation results [9]. Ensure that the scale of the interpolation control body is equivalent to that of the host unit, which helps to improve the interpolation accuracy [10]. In order to extend the application of unstructured overlapping grids to the case of multibody contact, researchers have studied the algorithm of unstructured overlapping grids with object contact, which further expands the application scope of unstructured overlapping grid technology [11].

Relevant scholars have extended the unstructured overlapping grid method to three-dimensional viscous flow simulation [8]. Although the subgrid is a hybrid grid containing tetrahedral and triangular prism elements when it is generated, the tetrahedral element is divided to obtain a unified tetrahedral mesh. Although this method has minor modifications to the original mesh assembly method and flow field solution, the element division itself is a complicated process, and the tetrahedral elements obtained by the division are often very long and narrow.

Relevant scholars have proposed that in addition to the traffic function, the street should have multiple functions and compound use [12]. They put forward the "street ethics" and "street eye" to lead the street back to the traditional space mode and create a more attractive public space. Related scholars have conducted research on people's behavior in the streets [13]. He opposed the streets of modern technologism and led the street space planning and design of some cities such as Melbourne. The practice has proved that pedestrianized and humanized urban streets are more dynamic. Both "Public Space.Public Life" and "New Urban Space" summarize and explain the practical results of urban street revival in European countries.

According to the different scales of research projects, the US Urban Design Guidelines often formulate and form guidelines at different levels, such as regional guidelines, city-wide guidelines, and regional guidelines [14]. In particular, for the design guidelines for large plots, the content of the guidelines mainly focuses on the development strategy, the development framework, and the grasp of the overall morphological characteristics; the design guidelines for smaller plots focus on controlling the environmental elements of the shape. The detailed design guidelines include environmental facilities such as street furniture, paving, and landscape signs [15].

The US Urban Design Guidelines compile corresponding guidelines for research objects in different regions and locations [16]. When formulating the design content, the Urban Design Guidelines do not blindly emphasize the comprehensiveness of the content but provide targeted design guidance for the different emphasis content of different types of design objects.
The content elements of the British city guide include bases, buildings, transportation, public spaces, greening and planting, materials and paving, lighting, facilities, signs, and other factors [17]. The design guide will be based on the different intrinsic attributes of the land. The different focus of the design is to select the main design elements. For example, in the design guide for Christchurch's new residential area, text descriptions and graphic demonstrations are provided for the three parts of site selection and planning, architectural design, and outdoor space design.

Singapore incorporated its urban design guidelines into development control regulations as the legal basis for design control $[18,19]$. The guidelines are coordinated with Singapore's master plan during the formulation process, integrating development control and design control, and during the operation of the guidelines, the public's needs and opinions and the developer's investment intentions are fully obtained in the form of consultation documents and exhibitions.

\section{Method}

2.1. Parallel Dual-Point Pair Aggregation AMG. In order to distinguish the multiscale and anisotropic characteristics between matrix units, it is necessary to define the strong and weak coupling relationship in the algebraic matrix. L-matrix $A$ is taken as an example, that is, the main diagonal element $a_{i j}$ is positive, and the nondiagonal element $a_{i j}$ is negative. The set of nodes that have a strong coupling relationship with matrix element $i$ is defined as follows:

$$
S_{i}=\left[a_{i j} \leq(1-\beta) \underset{a_{i k}>-1}{\max }\left|a_{i k}\right| \mid i \neq j\right] .
$$

Here, $\beta$ is the strong and weak coupling threshold constant, usually set to $\beta=0.21$. In a single aggregation, the two matrix units with the strongest coupling are selected for aggregation each time to form the solid unit of the next layer of the coarse grid. For the strongly diagonally dominant matrix nodes, it is generally considered that in the smooth iterative stage, the error elimination requirements have been met, and there is no need to restrict it to the lower grid. Here, the strongly diagonally dominant element can be defined as follows:

$$
D=\left\{a_{i j} \theta<\prod_{i \neq j}\left|a_{i j}\right| \mid i\right\} .
$$

Here, $\theta$ represents the strong diagonal dominance threshold, which is generally set as $\theta>5$.

Generally, the coarse-grid layer obtained by dual-point pair aggregation is used, the coarsening rate is low, and the calculation amount is relatively large. The two-point pair aggregation method is the two strongest coupling point pair aggregation algorithm, which increases the grid coarsening rate, reduces the amount of calculation at each level, and forms the next layer of coarse-grid matrix. The coarsening rate is defined as $n / n_{c}$, and the coarsening rate of the twopoint pair polymerization is less than 4 . When point-pair 
aggregation is used for the second time, the restriction of strong diagonal dominance is removed, and the strong diagonal dominance in multiple layers is only effective in the first layer.

For the coefficient matrix with multiscale and anisotropy, the strongest coupling point pair mode is adopted in both aggregation processes, and the matrix adjacency graph shows the combination of line modes in the strong coupling direction. It is a quadratic semi-coarsening process that satisfies the constraints of classical theory on grid coarsening for anisotropic problems.

\subsection{Geometric Characteristics of Aggregation Relations.} From an application perspective, GMG's calculation process strategy is relatively flexible, and the solution methods between each level can be independent of each other, using different iterative algorithms, and even the AMG convergence acceleration method can be used at each level of GMG. However, when GMG deals with complex problems such as complex shapes and high-extensibility grids, its efficiency is restricted to a certain extent. Algebraic equations are transformed from fluid problems on unstructured grids. For the original governing equations, using spatial and time discretization, linear system equations can be obtained, and physical problems are transformed into sparse linear equations. AMG takes the algebraic system matrix obtained by geometric discretization as the starting point. The construction of the matrix level is independent of physical problems and grid shape. The difference of GMG is that after constructing each level of the grid, it will be separately carried out on each grid level. When using the finite volume method to discretize, the calculation process must use the geometric features of the grid, such as volume, surface area, element center coordinates, and so on. Taking the implicit format two-layer multigrid as an example, the original governing equation after time discretization on the fine-grid layer $G_{f}$ can be expressed as follows:

$$
\left(\frac{\partial R_{i f}}{\partial Q_{i f}}-\frac{V_{i f}}{\Delta t} I\right) \Delta Q_{i f}-\frac{\partial R_{i f}}{\partial Q_{k f}} \Delta Q_{k f}=Q_{f} A_{f}
$$

When using AMG, the interpolation and restriction operators of the algebraic system are expressed as $I_{c f}$ and $I_{f c}$, respectively, and the error equation on the coarse-grid layer $G_{c}$ is as follows:

$$
A_{f} I_{f c} I_{c f}\left(A_{f} \Delta e_{f}\right)=R_{f} I_{f c} Q_{f} .
$$

At the same time, we use the error correction process to correct the results on the fine mesh layer:

$$
Q_{\text {new }, f}=I_{f c} Q_{f}-I_{c f} e_{c} \text {. }
$$

The algebraic coefficient matrix comes from the discreteness of the physical calculation grid, and any shape structure grid has a corresponding coefficient matrix structure. Figure 1 describes the mutual transformation relationship between unstructured grids and algebraic matrices.
2.3. Anisotropic Unstructured Grid. The difference in the geometric characteristics of the mesh is the main reason for the anisotropy in the numerical calculation process. Taking the finite volume discrete scheme as an example, this difference is mainly produced by surface integration or curve integration of the mesh. Generally speaking, when the ratio of the maximum area/minimum area (three-dimensional) or the maximum length/minimum length (two-dimensional) of the unit is larger, the anisotropic characteristics of the coefficient matrix will be more obvious.

The advantage of the node-centered calculation format in realizing the semiroughening method is that the extension line and the grid line are coincident, the extension line direction can be judged by the grid line, and it is not restricted by the geometric shape. There are two node template structures in this calculation format, namely, containment-dual tesselation (CDT) calculation template and median-dual tesselation (MDT) calculation template, as shown in Figure 2. CDT is obtained by connecting the center of the circumscribed circle. The advantage is that it is especially suitable for high-ductility mesh elements, which can effectively reduce the numerical dissipation error. The mesh extension direction is basically the same as the direction of the surface normal vector. The disadvantage is that the calculation efficiency is low. In comparison, MDT connects the center of mass of the shape to the midpoint of the edge and has a wider range of applications. However, on the triangular and tetrahedral meshes, the description of the anisotropy problem is not as intuitive as the CDT. The two calculation templates are consistent on the quadrilateral and hexahedral grids.

In order to reasonably describe the strong and weak coupling relationships in GMG, it is necessary to establish a weight map of the geometric grid and judge the strong and weak relationships of anisotropy by the difference of the weight value. In this study, the absolute value of the normal sum of the surface area of adjacent units is used as the criterion for anisotropy. When calculating a grid in two dimensions, this weight value represents the absolute value of the sum of the normal side lengths. This weight value is closest to the representation of the equation coefficients when the solution variable values between adjacent elements are very close. Therefore, the difference in its numerical value reflects whether it has anisotropic characteristics.

$w_{i j}$ is used to represent the weight value between the two units $i$ and $j$. If there is no adjacency between the two units, then $w_{i j}=0$. When the ratio of the maximum weight to the minimum weight in a unit exceeds a certain threshold $\beta$, the unit is considered to have anisotropic characteristics, and the adjacent unit with the largest weight value is regarded as the unit's strongest coupling adjacent unit. The unit set that has a strong coupling relationship with unit $i$ is defined as follows:

$$
S_{i}=\left\{w_{i j}<\Gamma \min w_{i k} \mid j \longrightarrow C\right\}
$$




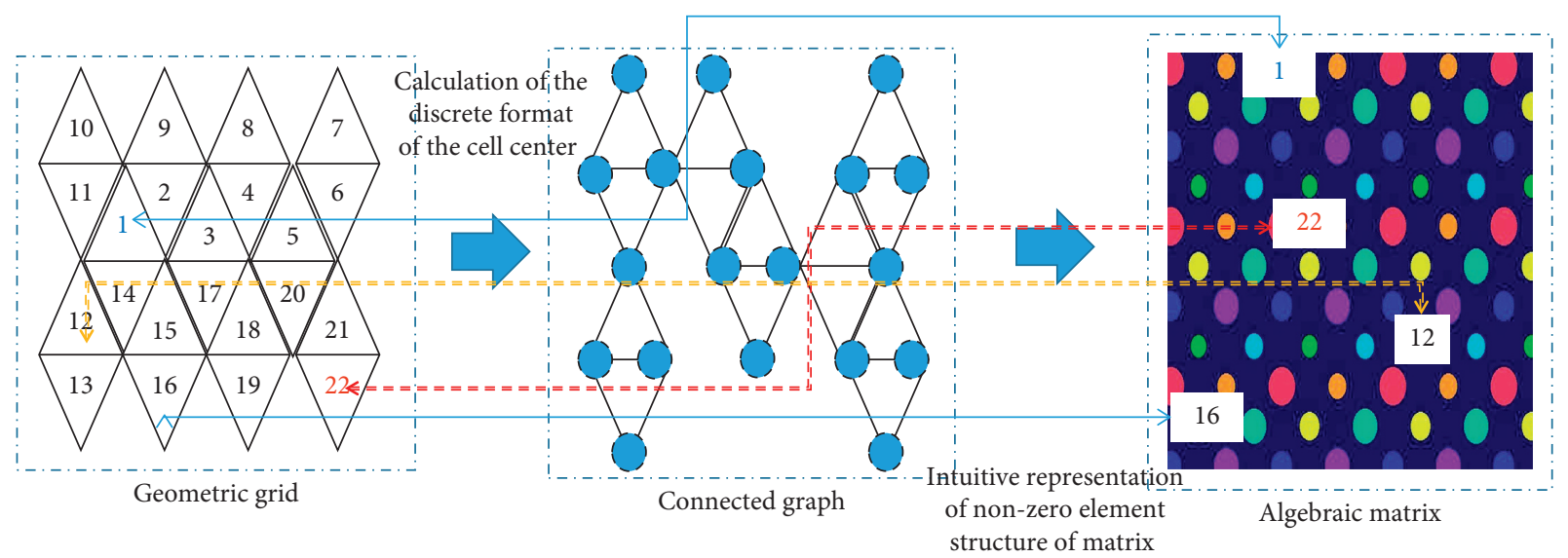

FIGURE 1: Inter-relationships among computational grids, matrix-connected graphs, and algebraic matrices.

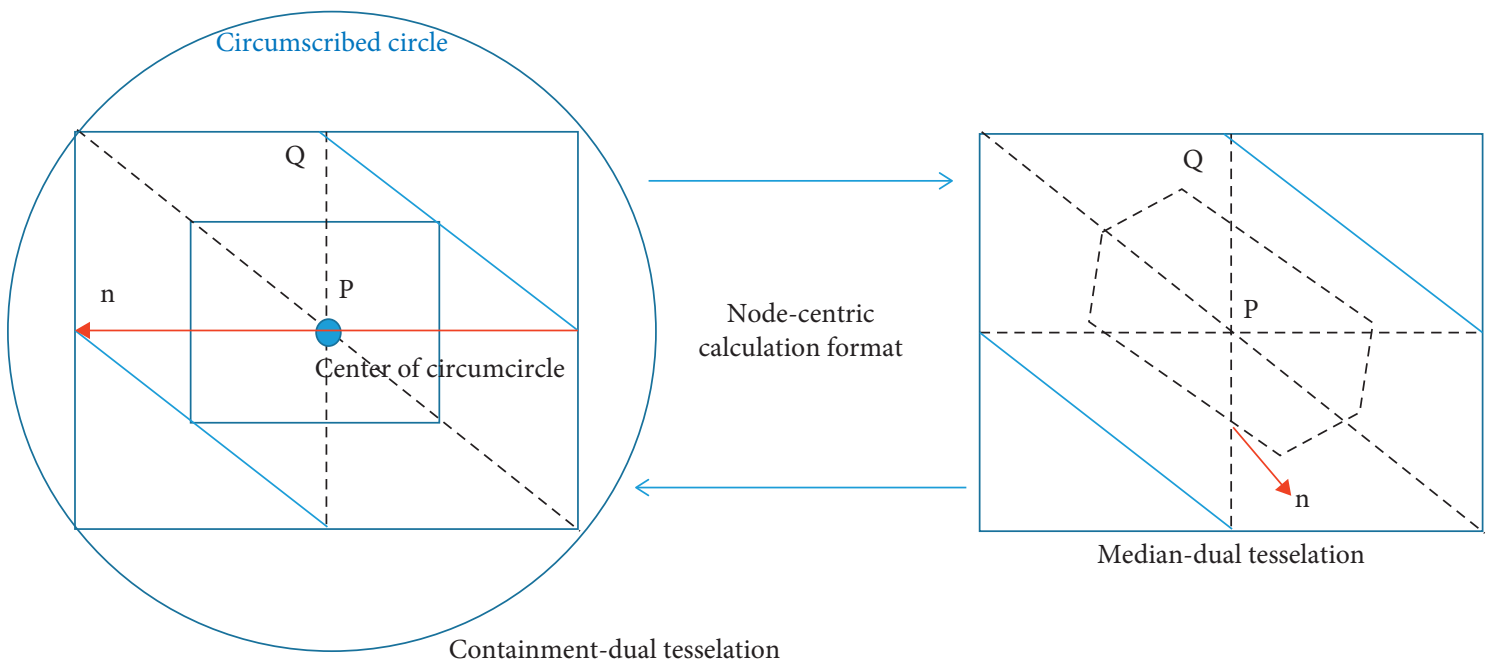

FIgURE 2: Comparison of unstructured grid node center-type discrete template.

The threshold $\Gamma$ represents the strong-weak coupling relationship and is generally set as $\Gamma=0.21$. For an isotropic grid cell, its adjacent cells are all strongly coupled cells; for anisotropic cells, only the adjacent cells that meet the conditions are called $i$ strongly coupled cells.

The AMG initial node selects the node with the least number of strongly coupled elements in the matrix row to reduce the number of comparisons in the aggregation process. The classic GMG aggregation algorithm usually adopts the order of the object surface boundary, the far-field boundary, and the internal unit. This algorithm uses the following sorting method:

$$
C=\left\{\frac{\min w_{i k}}{\max w_{i k}}<\frac{\min w_{j l}}{\max w_{j l}} \mid i, j ; k, l, i, j \longrightarrow C\right\} .
$$

The above sorting is based on the anisotropic elements generally concentrated on the boundary of the object surface and its affected area. From the point of view of numerical calculation, the greater the difference between the weight values, the more obvious the anisotropic grid characteristics, which need to be eliminated most during algorithm aggregation.
2.4. Improvement of the Transfer Operator. The limit operator is generally relatively fixed. In order to satisfy the conservation characteristics of the calculation equation, the solution vector limit operator uses a simple volume average, and the residual limit operator uses a simple superposition. No strategy has been found to improve the accuracy of limiting operators. The main reasons are reflected in the following three aspects:

(1) It is expensive to limit the increase in the accuracy of the operator. The limit operator uses the node template on the fine-grid layer, and the interpolation operator uses the node template on the coarse-grid layer. If the discrete accuracy of the limit operator is to be improved, the amount of calculation will significantly increase.

(2) The restriction operator only affects the numerical calculation on the coarse grid, and its discrete accuracy increases. Correspondingly, if the conservation characteristics of the control equation on the coarse-grid layer are satisfied, the node template on the coarse-grid layer must be expanded. At this time, 
it has the characteristics of overlapping grids, and the calculation amount is relatively large.

(3) The limit operator has no effect on error correction. The introduction of high-frequency errors often occurs in the process of error correction from the coarse-grid layer to the fine-grid layer, that is, the interpolation process. Even if the limit operator has higher discrete accuracy, the interpolation operator still uses direct assignment.

Geometric feature reconstruction expands the coarsegrid layer node template, and the idea of heuristic geometric weight is used to construct linear interpolation. Its expression is as follows:

$$
\Delta Q_{i f}=\frac{\Delta Q_{k c} \cdot I_{c f}}{\Delta Q_{l c} \cdot V_{n f}} .
$$

Here, $i$ represents a fine-grid unit, which is located in the $k$ unit of the coarse-grid layer, $N_{\text {if }}$ represents the set of units in the fine-grid layer that has an adjacent relationship with the $i$ unit, and the fine-grid unit $n$ is aggregated with adjacent units to form a coarse grid. Grid unit $l, V_{n f}$ is the volume of unit $n$. It can be seen that this interpolation operator expands the calculation node template to its neighboring units, with first-order discrete accuracy.

Aiming at the inaccuracy of the geometric feature reconstruction interpolation operator in the weight value distribution, this study reconstructs this interpolation operator, the node template used only modifies part of the weight ratio, and it also has the first-order discrete accuracy. The revised format is specifically expressed as follows:

$$
\Delta Q_{i f}=\frac{\Delta Q_{k c} \cdot V_{i f} \cdot I_{c f}}{\Delta Q_{l c} \cdot\left(V_{n f}+V_{i f}\right)} .
$$

\section{Result Analysis}

3.1. Evaluation of the G-R-D Model. The building area in the street reconstruction area of an urban area is $347,600 \mathrm{~m}^{2}$, and the average floor area ratio is 3.02. Among them, the residential building area is $323,800 \mathrm{~m}^{2}$, the average plot ratio is 2.81 , and the village collective economic property building area is $23,800 \mathrm{~m}^{2}$. The conditions before and after the transformation are shown in Table 1.

The resettlement of residential houses adopts a stepwise resettlement method, with four floors as the boundary. That is, if the legal infrastructure area is less than two floors, it is calculated as two floors. The original two floors and more than three floors are enough to compensate for three floors, the original three floors and more than four floors are enough to compensate for four floors, and the legal area with property rights is compensated for more than four floors. Residents can purchase the construction area beyond the original property rights area at a construction cost price of 3,500 yuan $/ \mathrm{m}^{2}$. Legal residences with four floors and below will be rebuilt in the same amount at a $1: 1$ ratio, and legal residences with four floors and above will be converted into commercial properties at a ratio of $2: 1$ and owned by the village collective. Operating financing funds must be earmarked for special purposes and strictly supervised.

In the process of street reconstruction in a certain urban area, the government played a leading role, issued policies and guidelines such as planning, taxes and fees, and property rights, coordinated various functional departments to assume consulting and service roles, and coordinated the transformation of a certain urban street. Under the vigorous promotion of the government, the demolition and reconstruction of a certain urban street eliminated the outdated image of the city such as the dilapidated handshake building and the poor sewage system. After the renovation is completed, professional property management is introduced, which greatly improves the management capabilities of the original villages, improves the quality of community management, and eliminates many potential safety hazards. The order of community management is gradually incorporated into the integrated urban management, and the level of integrated urban management is integrated. The image has been qualitatively improved. The economic benefits of the normalization of streets in a certain urban area are shown in Figure 3 .

3.2. Evaluation of the R-G-D Model. The characteristics of the R-G-D model are as follows: (1) under the guidance of the urban construction planning scheme, the residential form of the original residents has been completely changed, the building density is reasonable and standardized, and the community environment is more consistent with the urban environment. (2) The relocated houses are mainly used by residents themselves, and the overall construction volume will not be increased, which will not have an excessive impact on the real estate market. (3) The village collective self-raised funds for the construction of resettlement houses are for the residents' own use and not for sale. If the village collective has financing difficulties, it needs the policy support of the government. (4) Residents purchase resettlement houses at cost price. This model of both market and welfare has a great incentive effect on residents.

We invite 100 experts from government departments, street residents in a certain urban area, relevant personnel involved in street reconstruction in a certain urban area, stakeholders, and real estate development enterprises with experience in urban village reconstruction and use questionnaires to evaluate various types of urban renewal comprehensive benefit evaluation models. The indicators were evaluated, score data were collected, and 89 valid questionnaires were recovered. The statistical results of related data are shown in Figures 4 and 5.

Following the principle of economical and intensive use of land, the street reconstruction in an urban area adopts the practice of redesign and in situ reconstruction. The renovation and upgrading of each group have brought qualitative changes to the living environment and spatial environment of the streets in an urban area, perfected and enriched the functions of the new town, enriched the commercial value of the core area, optimized the urban landscape, and unified the modernity of the central area of the city. After the renovation 
TABLE 1: Statistics list of the status quo of streets in a certain urban area before reconstruction.

\begin{tabular}{lcc}
\hline & Project & \\
\hline Population & Household population & 6566 \\
& Migrant population & 9875 \\
Current total construction & Legal building & 45.5 \\
& Undocumented building & 3.7 \\
Total collective property volume & Legal collective supporting facilities & 4.9 \\
& Unlicensed collective supporting facilities & 1.6 \\
Total residential volume & Legal residence & 32.2 \\
& Undocumented residence & 2.8 \\
\hline
\end{tabular}

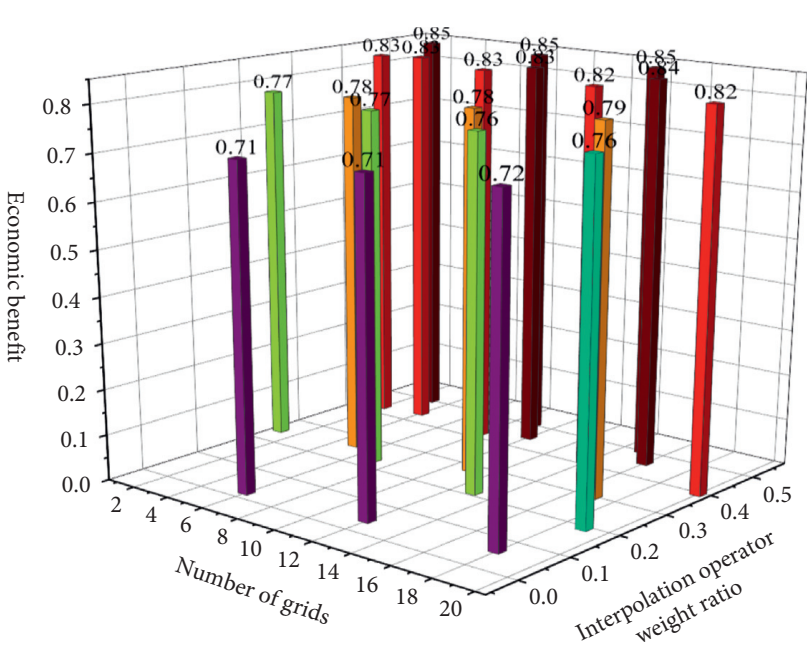

FIGURE 3: Economic benefits of normalization of streets in an urban area.

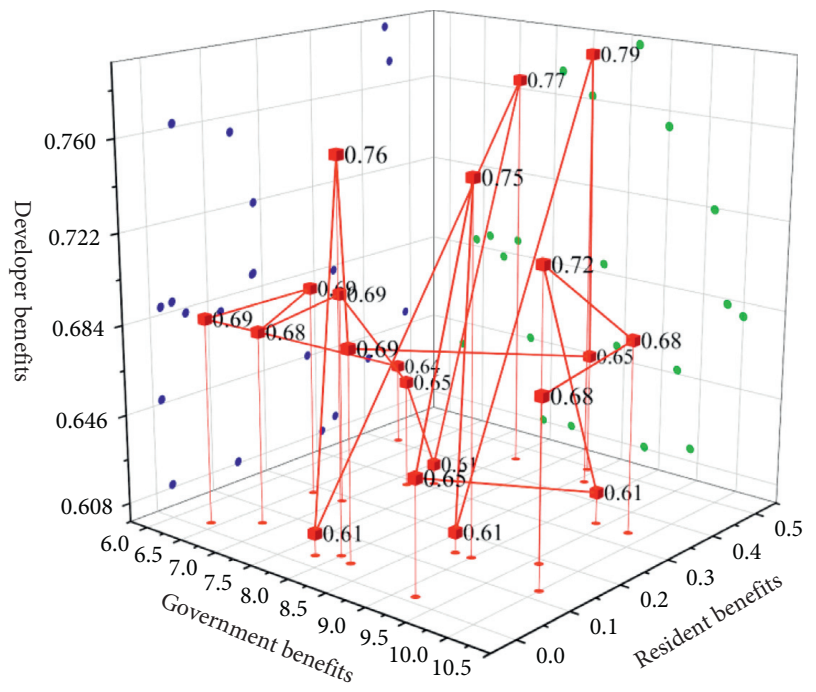

FIGURE 4: The relationship between government benefits, residents' benefits, and developers' benefits.

is completed, "a certain urban street" will be integrated into the city and transformed into an urban community integrating village collective structure, village collective economic organization, and clan relations. This is an urban-type residential area with a village collective nature. After the village reorganization, social administrative services such as old-age care, social insurance, and medical insurance in a certain urban area will be incorporated into the urban system, and urban governance will undergo positive changes.

After the transformation, a certain urban street collective will inherit the overall integrity of the elements of a specific community category under the specific historical environment of the "village." This is an urban residential area with the nature of a village collective. In order to continue the residents' memory of traditional villages and life, based on the traditional architecture of the original village, while retaining the structure and architectural style, it combines modern fashion elements and combines the antique commercial street with the Lingnan-style architecture of the Republic of China to form a unique style and artistic atmosphere.

3.3. Evaluation of the D-G-R Model. The total construction area of a street reconstruction area in an urban area is $681,903 \mathrm{~m}^{2}$, the total land area within the comprehensive renovation area is $758,000 \mathrm{~m}^{2}$, and the total planned construction area is 1.85 million $\mathrm{m}^{2}$, of which $750,000 \mathrm{~m}^{2}$ belong to collectives, including collective properties and relocation houses. The reconstruction area is divided into 13 plots. Among them, plots 6, 7, 8, and 9 are planned to be public green spaces with a total area of about $120,000 \mathrm{~m}^{2}$; plot 2 is a square land with an area of about $9,000 \mathrm{~m}^{2}$. Among the 1,3 , 4 , and 5 transfer plots, plot one is for residential and commercial finance, and the other three plots are for commercial finance; plot 11 is the construction land for rebuilding houses, including residential buildings, kindergartens, and vegetable markets; plot 12 is used as a reserve, with an area of $61541 \mathrm{~m}^{2}$; and plot 13 is construction land for primary and secondary schools. Figure 6 shows the proportion of construction plots for street reconstruction in an urban area.

In order to attract developers to participate in the renovation of a city's streets of $758,000 \mathrm{~m}^{2}$, the auction land price was only 3.56 yuan $/ \mathrm{m}^{2}$, and the total price was 142 million yuan. The developer bears the compensation for demolition and resettlement, the construction of relocation houses, and the construction of public infrastructure for the reconstruction of a certain urban street, which reduces the government's financial expenditure; it increases indirect income such as related taxes and fees for transactions with 


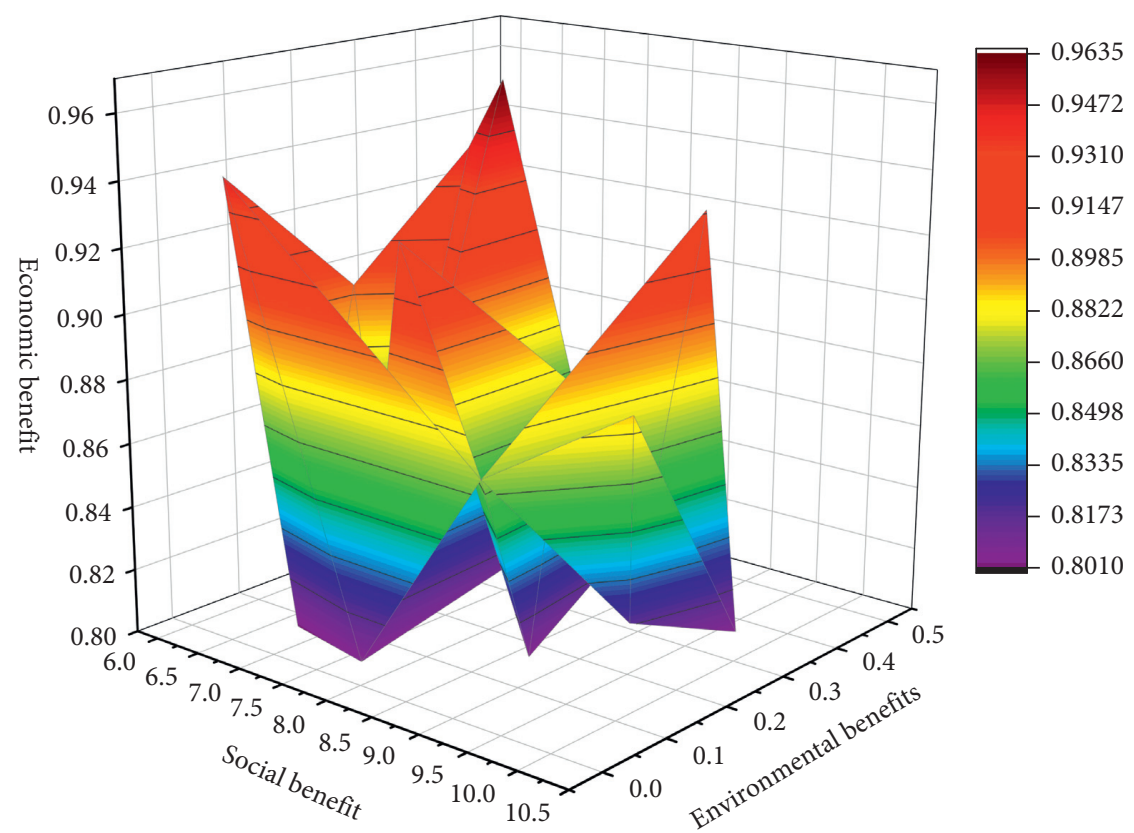

FIGURE 5: The relationship between social benefits, environmental benefits, and economic benefits.

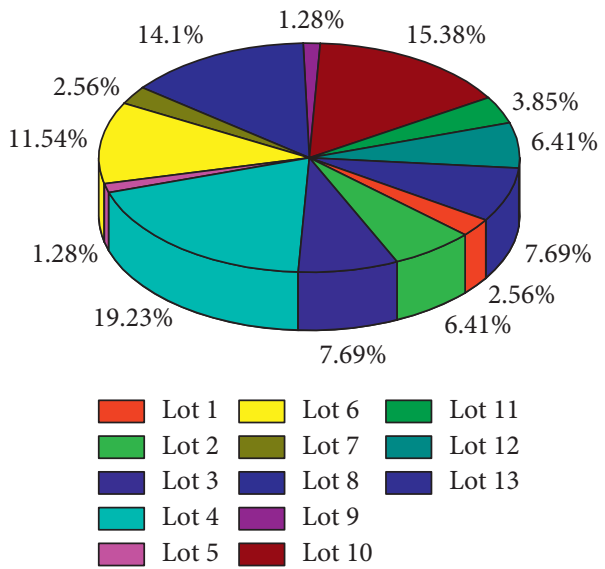

FIgURE 6: Proportion of construction plots for street reconstruction in an urban area.

TABle 2: The real estate income statement of each permanent resident in the street reconstruction of an urban area.

\begin{tabular}{|c|c|c|}
\hline Main economic benefits & $\begin{array}{l}\text { Compensation amount } \\
\text { (yuan) }\end{array}$ & Instruction \\
\hline $\begin{array}{l}\text { Compensation for undocumented } \\
\text { housing }\end{array}$ & 54500 & Unlicensed 1,000 yuan $/ \mathrm{m}^{2}$ material compensation \\
\hline Rental expenses & -60000 & 30 months average rent 18 yuan $/ \mathrm{m}^{2}$ \\
\hline Relocation subsidy & 8000 & Used for relocation subsidies \\
\hline Temporary resettlement compensation & 340000 & $\begin{array}{c}\text { Calculated an average of } 8700 \text { yuan per month based on } 30 \\
\text { months }\end{array}$ \\
\hline Early signing bonus & 7500 & Calculated as $50 \%$ of residents receive rewards \\
\hline
\end{tabular}

the developer. First, the government maintains public interests, not special interests. Second, a certain city government clarified that part of the land transfer fee should be allocated to the village collective economic organization and suggested that
$60 \%$ of the net income of the comprehensive renovation in the next five years should be allocated to the village collective economy. The district government sets up special funds to support the development of a certain urban street and improve 


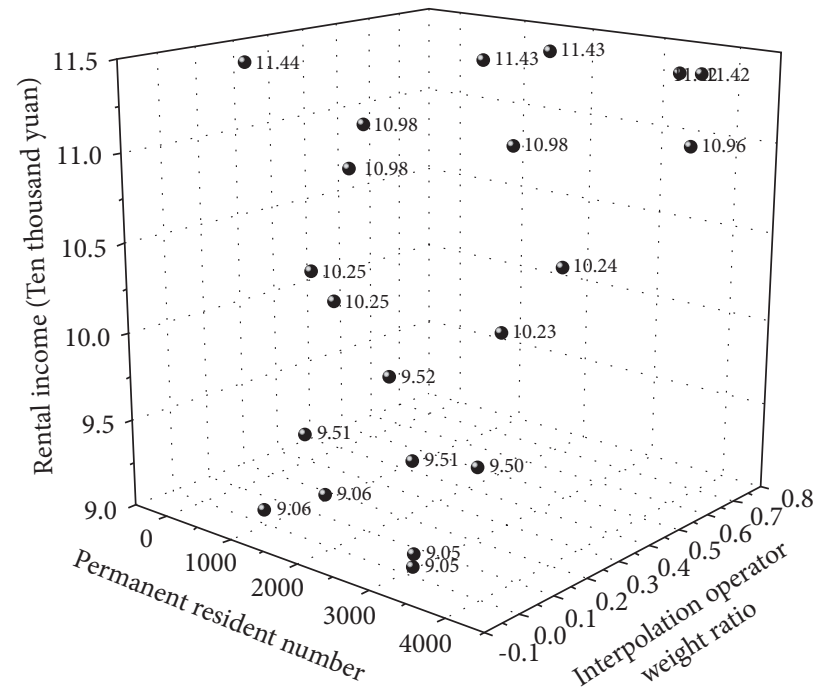

FIGURE 7: Every permanent resident in a certain urban district will evenly share the real estate income without renovation.

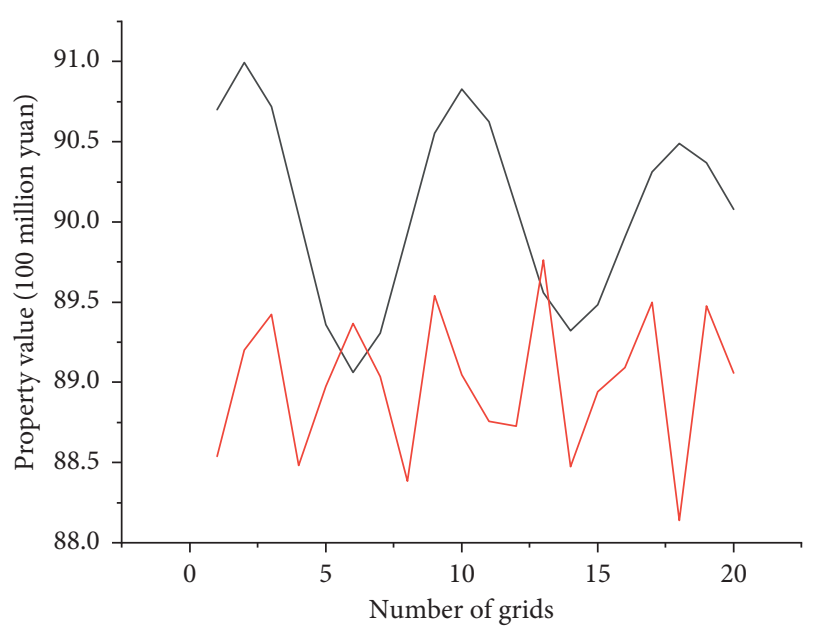

- Lot one

- Lot five

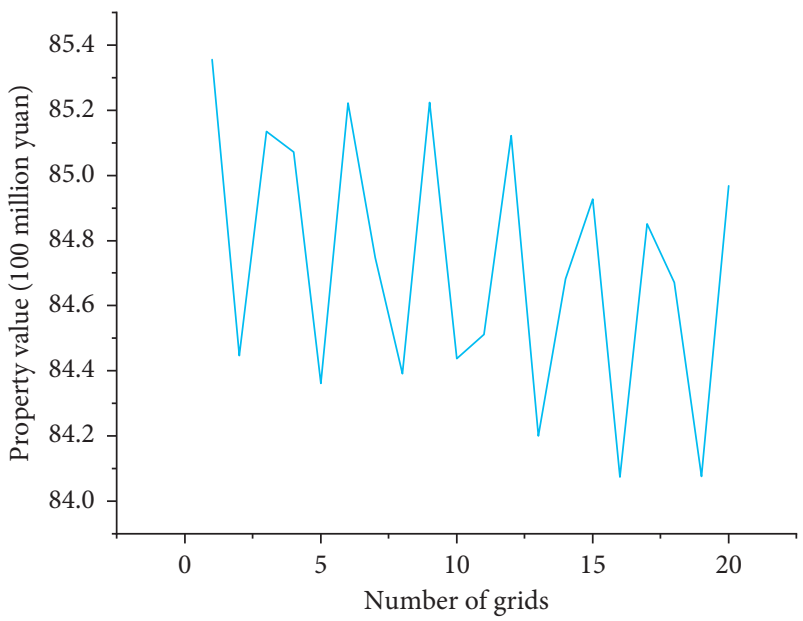

__ Lot three

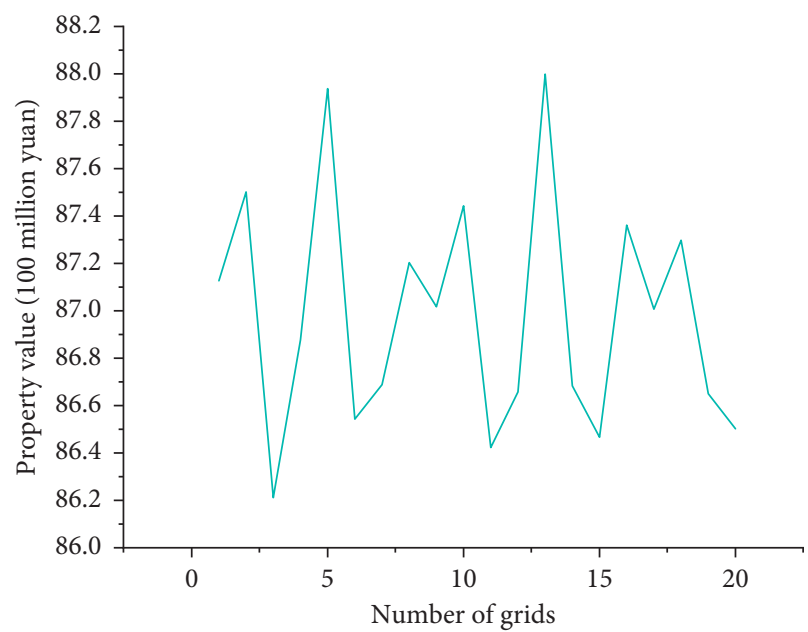

— Lot two

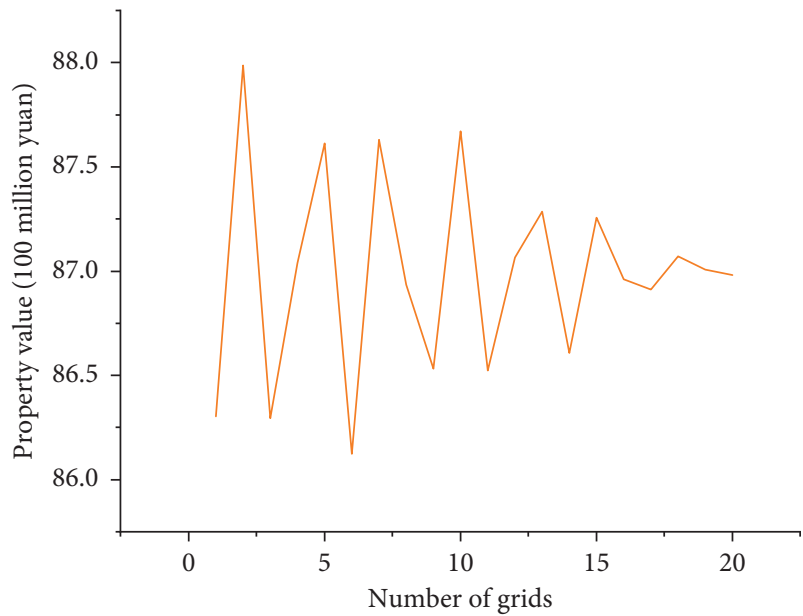

_ Lot four

Figure 8: Real estate value created by developers in the street reconstruction of an urban area. 
public buildings and municipal public infrastructure in the surrounding areas. Table 2 shows the average share of real estate income for each permanent resident in the street reconstruction of an urban area. Figure 7 shows the average share of real estate income for each permanent resident in a certain urban street without reconstruction.

The developer participates in the street reconstruction of a certain urban area and is responsible for the demolition and overall reconstruction of the entire large-scale system project. In addition to enhancing social reputation and establishing a corporate image, the more important enterprise expectation is to obtain considerable capital operation benefits. First, the floor area ratio of the resettlement area approved by the government is 4.72 . Second, in the fourth plot for commercial development by developers, it is planned to build an ultra-high building with more than 60 floors and a height of $200 \mathrm{~m}$, with a plot ratio of 6.07 .

The total investment of a street reconstruction project in an urban area is about 17 billion yuan, of which about 5.5 billion yuan will be spent on the demolition and resettlement of residents and the construction cost of relocation houses, about 300 million yuan will be spent on the renovation and renovation of the ancient port of Huangpu, and about 3 billion yuan will be built for infrastructure such as municipal and greening. The remaining funds are used for commercial development and construction. The main income of its developers comes from the commercial development of plots 1, 3, 4, and 5. Through the "Street Reconstruction Plan and Constructive Detailed Planning of a Certain Urban District," it is possible to analyze the approximate economic benefits of the developer in the process of street reconstruction in a certain urban area. From the perspective of profit maximization, the developers have gained considerable benefits from the renovation of a certain urban street in three years. Figure 8 shows the real estate value created by developers in the street reconstruction of a certain urban area.

\section{Conclusions}

In view of the fact that unstructured grid aggregation is greatly affected by grid type and physical structure, especially the defects of high-ductility elements that cannot be handled well, this study is based on the analysis of the characteristics of nonsmooth aggregation of algebraic multigrid. The anisotropic geometric features are transformed into numerical relationships, the strong and weak geometric coupling relationships are distinguished by their sizes, and a strong coupling aggregation strategy is developed. The aggregation method compares the strong and weak coupling relationships of the grid to determine whether it is a highly ductile unstructured grid unit and separately treats the aggregation of the two types of units, which is well adapted to the fluid calculation of two-dimensional and three-dimensional unstructured grids. We discussed the connotation of the comprehensive benefit evaluation of urban renewal and designed two evaluation systems of coordination benefit and development benefit to comprehensively evaluate the comprehensive benefit of urban renewal. The coordination benefit system includes government benefit, resident benefit, and developer benefit, and the development benefit system includes social benefit. Through literature research and questionnaire surveys, 30 representative influencing factors for comprehensive benefit evaluation of urban renewal were determined. Using factor analysis, a comprehensive benefit evaluation index system for three urban renewal models was constructed. The goals of urban renewal are multidimensional, and its comprehensive benefit evaluation must be not only evaluated from the development goal elements of society, economy, and environment but also from the perspective of the coordination of the interests of the government, residents, and developers of stakeholders. People's livelihood, ecology, culture, and economy are the core content of the comprehensive benefits of urban renewal. The social and people's livelihood is the foundation of urban development. The ecological environment is an important factor in the evaluation system. Economic development is an important condition for urban development. The balance of interests of stakeholders is urban renewal. For urban renewal, it is necessary to comprehensively consider the coordinated and sustainable development of society, environment, and economy. The research results of this study have certain reference value and practical guiding significance for the harmonious and sustainable development of urban renewal in our country. When formulating relevant policies, the government can comprehensively consider the urban renewal plan from multiple perspectives of evaluation elements, quantify the comprehensive benefits through the comprehensive benefit calculation method, select the transformation plan with the best comprehensive benefit, and strive to achieve the goal of urban renewal and sustainable development.

\section{Data Availability}

The data used to support the findings of this study are available from the author upon request.

\section{Conflicts of Interest}

The author declares no conflicts of interest.

\section{Acknowledgments}

This work was supported by Xi'an Fanyi University.

\section{References}

[1] A. Jamshidnejad, I. Papamichail, M. Papageorgiou, and B. De Schutter, "Sustainable model-predictive control in urban traffic networks: efficient solution based on general smoothening methods," IEEE Transactions on Control Systems Technology, vol. 26, no. 3, pp. 813-827, 2018.

[2] T. Jiang, D. Hu, and X. Yu, "Enhanced IT2FCM algorithm using object-based triangular fuzzy set modeling for remotesensing clustering," Computers \& Geosciences, vol. 118, pp. 14-26, 2018.

[3] Y. Huang, N. Guo, M. Seok, Y. Tsividis, and S. Sethumadhavan, "Evaluation of an analog accelerator for 
linear algebra," ACM SIGARCH - Computer Architecture News, vol. 44, no. 3, pp. 570-582, 2016.

[4] R. Arghandeh, A. von Meier, L. Mehrmanesh, and L. Mili, "On the definition of cyber-physical resilience in power systems," Renewable and Sustainable Energy Reviews, vol. 58, pp. 1060-1069, 2016.

[5] Y. Wang, D. Wang, S. Jin, N. Xiao, Y. Li, and E. Frazzoli, "Iterative tuning with reactive compensation for urban traffic signal control," IEEE Transactions on Control Systems Technology, vol. 25, no. 6, pp. 2047-2059, 2017.

[6] S. I. Toure, D. A. Stow, H.-c. Shih, J. Weeks, and D. LopezCarr, "Land cover and land use change analysis using multispatial resolution data and object-based image analysis," Remote Sensing of Environment, vol. 210, pp. 259-268, 2018.

[7] J. Zhang, Z. Wang, and N. Verma, "In-memory computation of a machine-learning classifier in a standard 6T SRAM array," IEEE Journal of Solid-State Circuits, vol. 52, no. 4, pp. 915-924, 2017.

[8] S. C. Zipper, M. Motew, E. G. Booth et al., "Continuous separation of land use and climate effects on the past and future water balance," Journal of Hydrology, vol. 565, pp. 106-122, 2018.

[9] Y. Xu, "Hybrid jacobian and gauss--seidel proximal block coordinate update methods for linearly constrained convex programming," SIAM Journal on Optimization, vol. 28, no. 1, pp. 646-670, 2018.

[10] T. Chen, A. Sun, and R. Niu, "Effect of land cover fractions on changes in surface urban heat islands using Landsat timeseries images," International Journal of Environmental Research and Public Health, vol. 16, no. 6, p. 971, 2019.

[11] L. Lin, C. Jiang, X.-D. Xu, Y.-L. Guo, and T. Chen, "Study on evaluation for crowd degree of traffic flow at urban tunnel access based on acceleration noise," Journal of Highway Transp. Res. Develop. (English Ed.), vol. 11, no. 1, pp. 70-76, 2017.

[12] Y. Tian, W. Hu, B. Du, S. Hu, C. Nie, and C. Zhang, "IQGA: a route selection method based on quantum genetic algorithmtoward urban traffic management under big data environment," World Wide Web, vol. 22, no. 5, pp. 2129-2151, 2019.

[13] J. Wang, S. Zhang, and Y. Guo, "Analyzing the impact of impervious area disconnection on urban runoff control using an analytical probabilistic model," Water Resources Management, vol. 33, no. 5, pp. 1753-1768, 2019.

[14] L. Qi, M. Zhou, and W. Luan, "A dynamic road incident information delivery strategy to reduce urban traffic congestion," IEEE/CAA Journal of Automatica Sinica, vol. 5, no. 5, pp. 934-945, 2018.

[15] X. Cao, X. Gao, Z. Shen, and R. Li, "Expansion of urban impervious surfaces in Xining city based on GEE and Landsat time series data," IEEE Access, vol. 8, pp. 147097-147111, 2020.

[16] W. Xiangxue, X. Lunhui, and C. Kaixun, "Data-driven shortterm forecasting for urban road network traffic based on data processing and LSTM-RNN," Arabian Journal for Science and Engineering, vol. 44, no. 4, pp. 3043-3060, Apr. 2019.

[17] F. Huang, Y. Yu, and T. H. Feng, "Automatic extraction of impervious surfaces from high resolution remote sensing images based on deep learning," Journal of Visual Communication and Image Representation, vol. 58, pp. 453-461, 2019.

[18] R. Al Mallah, A. Quintero, and B. Farooq, "Cooperative evaluation of the cause of urban traffic congestion via connected vehicles," IEEE Transactions on Intelligent Transportation Systems, vol. 21, no. 1, pp. 59-67, 2020.

[19] S. Feng and F. Fan, "A hierarchical extraction method of impervious surface based on NDVI thresholding integrated with multispectral and high-resolution remote sensing imageries," Ieee Journal of Selected Topics in Applied Earth Observations and Remote Sensing, vol. 12, no. 5, pp. 1461-1470, 2019. 